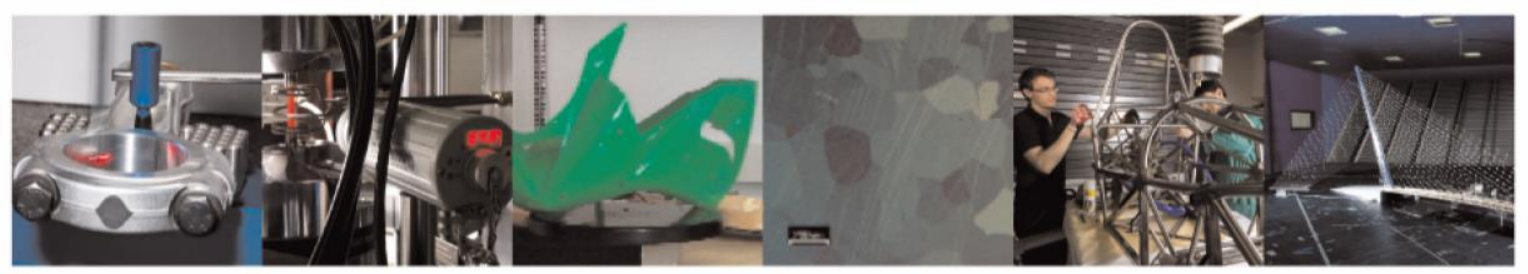

\title{
Real-Time Observation of Melt Pool in Selective Laser Melting: Spatial, Temporal, and Wavelength Resolution Criteria
}

Mazzoleni L.; Demir A.G.; Caprio L.; Pacher M.; Previtali B.

This is a post-peer-review, pre-copyedit version of an article published in IEEE Transactions on Instrumentation and Measurement. The final authenticated version is available online at: http://dx.doi.org/10.1109/TIM.2019.2912236

This content is provided under $\underline{\text { CC BY-NC-ND } 4.0}$ license

\section{(c) (1) (9)}

(C) 20XX IEEE. Personal use of this material is permitted. Permission from IEEE must be obtained for all other uses, in any current or future media, including reprinting/republishing this material for advertising or promotional purposes, creating new collective works, for resale or redistribution to servers or lists, or reuse of any copyrighted component of this work in other works. 


\title{
Real-Time Observation of Melt Pool in Selective Laser Melting: Spatial, Temporal and Wavelength Resolution Criteria
}

\author{
Luca Mazzoleni, Ali Gökhan Demir, Leonardo Caprio, Matteo Pacher, and Barbara Previtali
}

\begin{abstract}
Melt pool monitoring in selective laser melting (SLM) is a key challenge to enhance the understanding of the basic process physics as well as the in-situ identification of drifts. The main issues are related to its fast dynamics and small spatial extent, which require high performance monitoring systems in terms of temporal and spatial resolution, often resulting in unmanageable data rates. Furthermore, the broadband thermal emission from the process can be viewed in different spectral ranges depending on the sensor and optical system employed. Accordingly, a wide range of sensing configurations is possible. The choice of monitoring parameters is crucial to achieve a solution capable of describing process dynamics with industrial applicability in terms of data transport and analysis. This work discusses the design of a coaxial monitoring module to assess melt pool dynamics in SLM using temporal and spatial cues related to the process physics. Restrictions regarding data management were considered to ensure a future inline process control. The system was tested with an external illuminator to reveal the actual melt pool geometry employing an acquisition rate of 1200 fps, $4.3 \mathrm{~mm} \times 4.3 \mathrm{~mm}$ field of view and $14 \mu \mathrm{m} / \mathrm{pixel}$ spatial resolution. The process emission in visible $(640 \mathrm{~nm})$ and nearinfrared regions $(850-1000 \mathrm{~nm})$ was also acquired and the band choice discussed. The proposed solution captured successfully the melting conditions from both a spatial and temporal viewpoint. The monitoring system depicted variations of the melt pool shape when processing different geometries using modulated and continuous wave laser emission.
\end{abstract}

Index Terms-Selective laser melting, process monitoring, thermal emission, infrared imaging, melt pool geometry.

\section{INTRODUCTION}

$\mathrm{S}_{\mathrm{B}}^{\mathrm{B}}$ ELECTIVE laser melting has been gaining importance in $\checkmark$ the industrial scenario, enabling a full-scale production of complex metal components. Nonetheless, there are still open aspects preventing the SLM manufacturing technology from achieving its full potential and diffusion. One major problem is the quality and reproducibility of produced parts. During the SLM process different errors can occur, namely incorrect

This work was supported by European Union, Repubblica Italiana, Regione Lombardia and FESR for the project MADE4LO under the call "POR FESR 2014-2020 ASSE I - AZIONE I.1.B.1.3".

The authors are with the Mechanical Department of Mechanical Engineering, Politecnico di Milano, Via La Masa 1, 20156 Milan, Italy (email: luca.mazzoleni@polimi.it; aligokhan.demir@polimi.it; leonardo.caprio@polimi.it; barbara.previtali@polimi.it). powder spread [1], internal defects such as incomplete melting or pores [2], and even part deformation and cracking [3] due to high temperature gradients within the process. Detecting process anomalies during the powder melting is highly desired in order to undertake corrective actions as soon as they are detected, therefore avoiding time-consuming and costly quality inspections [4]. Furthermore, the lack of knowledge on the SLM process physics represents a major point of weakness in its development. To date, analytical models and numerical simulations are still being developed with the aim to provide an exhaustive description of the complex physical mechanisms involved in such a dynamic process. Such models require experimental inputs also for calibration and validation [5]. Consequently, SLM's progress still relies strongly on experimental trials and heuristic approaches. Nevertheless, a deeper understanding of SLM can be highly beneficial for the improvement of this promising technology.

To overcome some of these challenges, much emphasis has recently been placed on the observation of SLM melting conditions. Numerous research efforts address the monitoring and control of SLM with different instruments, setups and targets in order to gather information on process stability and to enhance the fundamental understanding of the underlying physics. Currently the research for an effective process control solution remains an open question [6]. The fast dynamics of the laser beam across the powder bed and the small size of the laser interaction zone make the observation of the process a challenging task, since both high spatial resolution and temporal details are necessary at the same time to assure a proper visualization of the melting conditions [7]. Spatially integrated sensors, such as photodiodes and pyrometers, are widely employed in SLM monitoring thanks to their fast response and easy integration; they both provide acquisition rates above $50 \mathrm{kHz}$, are low cost and generate a low amount of data [8]-[12]. On the other hand, the integrating nature of these sensors limits considerably the content of the information acquired, preventing the extraction of geometrical indicators of the melting conditions. An alternative approach to monitor the melting process is to employ spatially resolved sensors, i.e. cameras. High speed cameras are used in a coaxial [13]-[15] or in a lateral configuration [16], [17] to acquire the emission from the process in visible and in the NIR region up to $1 \mu \mathrm{m}$ wavelength. Also the mid or long-wave infrared region can be investigated through the use of an IR camera 
with a germanium sensor, usually from an off-axis perspective [18]-[22]. Regardless of the wavelength range of interest, spatially resolved sensors suffer from the generation of a massive amount of data (a matrix of data values is generated for each acquisition instant), which can be difficult to handle in real-time during the whole fabrication of a part. As a consequence, this promising monitoring method is often employed in phenomenological studies [23]-[27], where specific process conditions are observed by means of a high temporal resolution camera $(20 \mathrm{kHz}$ or more) for a limited acquisition time, no more than a few seconds, with data amounts reaching easily the gigabyte per second range. Some research efforts address the use of cameras within manageable data rates to monitor the melting conditions in a whole building process. However, the spatial resolution and the temporal resolution are in turn sacrificed in order to attain a lower amount of data. For example, Krauss et al. [28] capture the thermal emission of the laser processing zone by means of a long wave infrared camera with an acquisition rate limited to $50 \mathrm{fps}$, which does not really capture the melting process dynamics, since the laser beam scanned several hatch lines between each frame. Berumen et al. [7] reached $10 \mathrm{kHz}$ acquisition rate, but the spatial resolution was limited by the use of a $20 \times 16$ pixels window. While optical sensors are sensitive only to surface events, in situ X-ray imaging proved to be a powerful tool to investigate sub-surface melting mechanisms. Nevertheless, the highly complex architecture needed to capture the bulk material behavior limited the application only to phenomenological studies of predefined process conditions [29]. Recently, industrial SLM systems have adopted optical monitoring modules for part quality assurance. Concept Laser QM modules (Concept Laser $\mathrm{GmbH}$, Lichtenfels, Germany) provides a camera capturing an observation area of $1 \mathrm{~mm}^{2}$ with an acquisition rate of $15 \mathrm{kHz}$ and a spatial resolution of $35 \mu \mathrm{m}$ per pixel. Furthermore, a photodiode was implemented to acquire the IR radiation emitted from an area of $4 \mathrm{~mm}^{2}$ with a temporal resolution of $50 \mathrm{kHz}$ [30]. Renishaw plc (InfiniAM Spectral, Renishaw plc, Wotton-under-Edge) provides two photodiodes acquiring the emission from the process in visible and the IR range at 100 $\mathrm{kHz}$. The choice of different monitoring parameters highlights the need for a deeper understanding of the design criteria. In particular, further attention is required to identify the sensor type and its temporal, spatial, and wavelength resolution specifications, which determine the final capability of the system to gather relevant information on the process and its industrial applicability in terms of data rate generated.

The aim of the work presented herein is to provide new insights in the design of a monitoring module for melt pool measurement in SLM. In particular, this work analyses the limiting criteria for spatial, temporal resolutions as well as the selection of the observation wavelength band together. A spatially resolved sensor, a CMOS camera, was chosen to

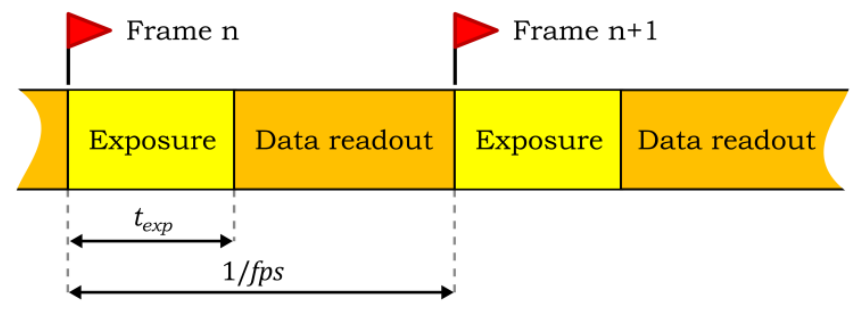

Fig. 1. Camera acquisition rate and exposure time.

gather spatial information on the melt pool. Physical phenomenon characteristics, both in the temporal and spatial domain, were analyzed and a monitoring module for melt pool observation was designed according to the process physics requirements. The acquisition of useful information in time and space within a manageable data rate in terms of real-time data transport allowed to monitor a whole SLM building without limitations on the duration of the recording. Once the camera sensor and the camera lens have been chosen, optical filters must be carefully chosen to focus on the wavelength bands of interest. In this work, the process emission in visible and in NIR wavelength bands were acquired and the choice of the emission band is discussed. Furthermore, an external illumination was employed to dominate the emission from the process, thus enabling the observation of the real melting conditions and a direct interpretation of the signals acquired. The melting conditions were observed and compared when realizing different geometries (a bulk region and a thin structure) and varying the emission mode of the laser source at a fixed energy density (continuous wave and pulsed wave). The comparison between the direct visualization of the melt pool geometry and the process emission showed that the emitted radiance in the NIR range with an adequate calibration could successfully represent the molten pool geometry.

\section{Design Criteria for the Monitoring Module}

When designing an imaging system for process visualization, several design parameters should be taken into account. They can be listed as follows.

- Temporal resolution determines the capability of the system to capture the dynamic behavior of the object under inspection. Two parameters affect the system resolution in time: the camera frame rate $f p s$ (frame per second), and its exposure time $t_{\text {exp }}$, i.e. the temporal interval during which the sensor is exposed to light, thus integrating the entire incoming radiation. The exposure time can be equal or lower than the time interval between two consecutive frames $1 / f p s$, as schematically illustrated in Fig. 1.

- Spatial parameters, namely spatial resolution and field of view, are fundamental in order to visualize correctly the process signature of interest and avoid data burden. The spatial resolution $r_{s}$, usually expressed as $\mu \mathrm{m} /$ pixel, determines the capability of the monitoring system to resolve object spatial details. Since the image of the object under inspection is discretized into a matrix of grey levels corresponding to the pixels composing the 


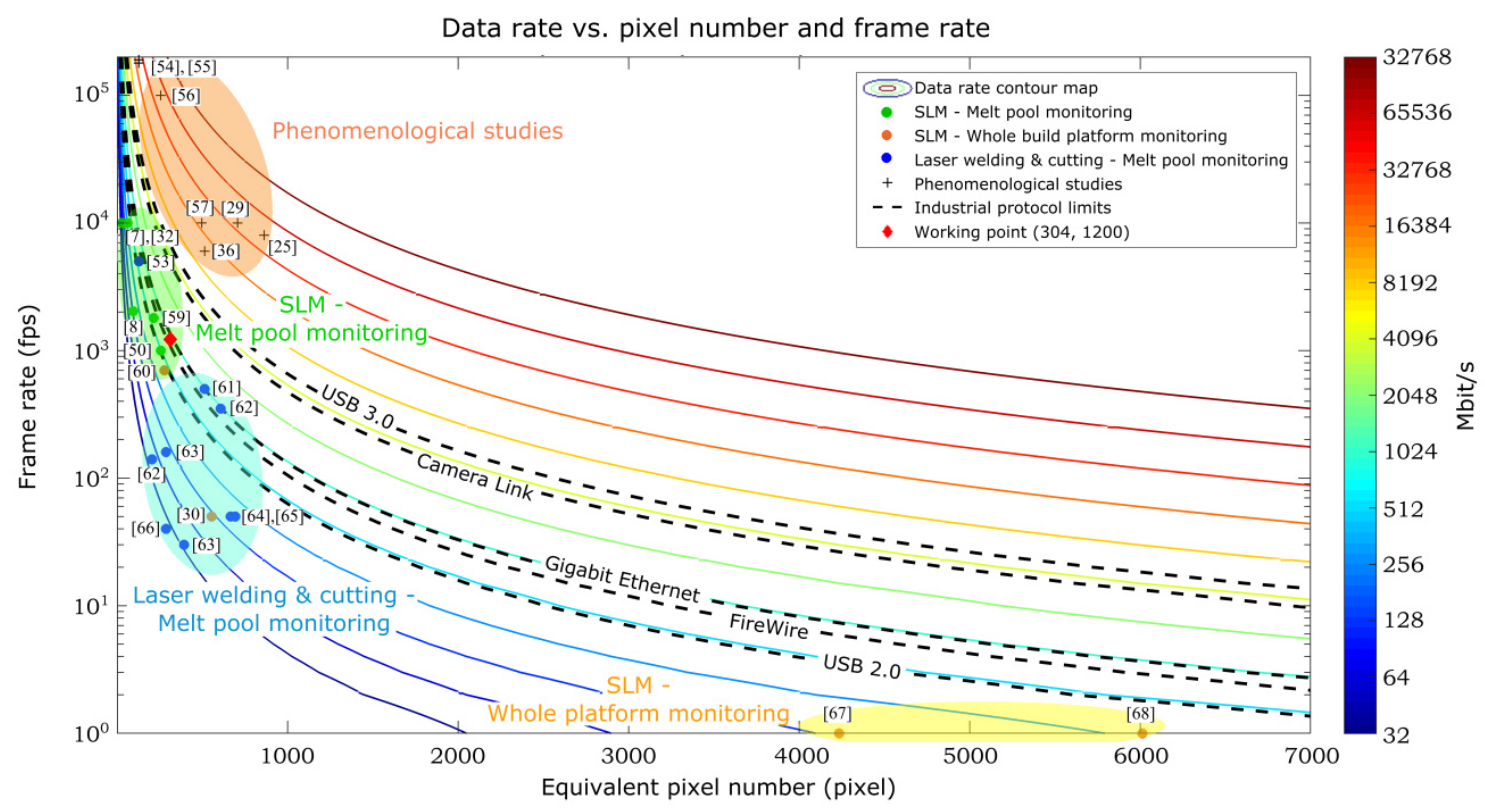

Fig. 2. Literature review of monitoring and phenomenological studies on SLM as well as laser welding and cutting (x axis: equivalent pixel number calculated as the square root of the total number of pixels employed, y axis: acquisition rate). Different operating area are individuated and highlighted by specific colors.

camera sensor, the minimum feature size of the object detectable by the sensor depends both on camera pixels size $s_{p}$ and on magnification $m$ of the object under investigation given by the optical system according to the following equation:

$r_{s}=s_{p} / m$

Eq. (1) shows the importance of a proper choice of the spatial resolution for an imaging system, since it has an impact on both the choice of the sensor and the selection of the optical lenses. Once spatial resolution has been fixed, the field of view $F_{x}(\mathrm{~mm}) \times F O V_{y}(\mathrm{~mm})$ should be chosen, which defines the extent of the area observed both horizontally and vertically. The number of pixels $n_{p, x}$ needed to assure the desired $F O V_{x}$ can be calculated for the horizontal direction as:

$n_{p, x}=F O V_{x} / r_{s}$

The number of pixels in the vertical direction $n_{p, y}$ can be evaluated in an analogous way to $n_{p, x}$ :

$n_{p, y}=F O V_{y} / r_{s}$

- All the aforementioned parameters affect the amount of data generated per unit time $R_{b}(b i t / s)$ according to the following equation:

$R_{b}=n_{p, x} \cdot n_{p, y} \cdot b d \cdot f p s$

where $n_{p, x} \cdot n_{p, y}$ is the total number of pixels employed, $f p s$ is the camera acquisition rate and $b d$ is the bit depth determining the analog-digital conversion resolution expressed as bit/pixel. In order to assure a real-time acquisition of data and the transport towards an elaboration unit, the data rate should not exceed the data transfer rates allowed by common digital camera interfaces (for example, theoretical limits for USB3.0 and GigE are $5 \mathrm{Gbit} / \mathrm{s}$ and $1 \mathrm{Gbit} / \mathrm{s}$ respectively). Fig. 2 shows the data rate as a function of the frame rate $f p s$ and the number of pixels employed. A semilogarithmic scale was used, and each colored line represents the locus of points with equal data rate amount as indicated in the color scale. The different digital interface limits are depicted with black dotted lines. The graph illustrates different monitoring and diagnostic solutions used in the literature for SLM as well as laser cutting and welding. A first clear distinction between monitoring and diagnostic approaches can be noticed. Phenomenological studies show a data rate well above the limits imposed by industrial interface protocols, thus confirming the significant data amount necessary to look at the very heart of process physics. Conversely, monitoring strategies make use of a compromise between the number of pixels and the acquisition rate in order not to overcome data transport limits. Accordingly, lateral solutions with a field of view extended to the whole build platform use more pixels at lower acquisition rate. Whereas, monitoring strategies with a smaller field of view focused on the melt pool feature a reduced number of pixels at a comparable spatial resolution, as highlighted by (2) and (3). This enables higher acquisition frequencies. In fact, if the number of pixels employed is lower - i.e. a reduced field of view and an adequate spatial resolution are chosen - higher acquisition frequencies are possible within data rate 


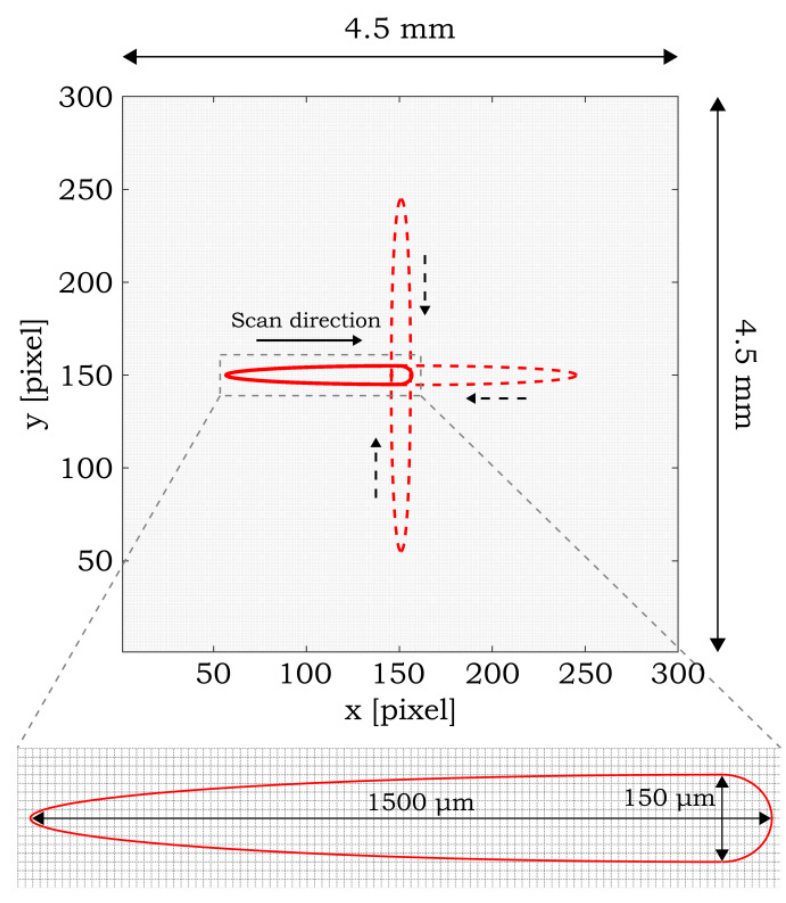

Fig. 3. Schematic representation of the final FOV and spatial resolution for melt pool imaging. The dotted red lines represent the molten pool orientation corresponding to different scan directions (black dotted arrows).

limits according to (4). In particular, melt pool observation in SLM shows higher acquisition frequencies than laser welding or cutting, where the processing speeds involved are significantly lower. This highlights the demanding requirements for SLM in terms of the acquisition rate needed to capture the fast dynamic of the melting process, decreasing the exploitable amount of pixels to ensure real-time data transport (top-left position in the map).

- The wavelength band of interest represents a critical issue for a successful process visualization. Spectral filters must be carefully chosen to focus on a predefined wavelength band in order to reduce chromatic aberration and to focus on different physical phenomena occurring during the powder melting process [31].

In order to acquire useful information and avoid data burden, the monitoring system design should be based on physical phenomenon requirements according to the following criteria.

\section{A. Temporal resolution criteria}

The dynamic behavior of the melt pool determines the frequency of the acquisition needed to capture its evolution in time. Some of the key physical phenomena known from laser welding as well as the SLM process are related to balling formation, keyhole dynamics and the melting phenomenon itself. All the frequencies associated with the melt pool activity are in $\mathrm{kHz}$ range, as reported in Table I.

Furthermore, the melt pool moves with a certain velocity on the build platform according to the scan speed $v$ employed
TABLE I

MELT POOL RELATED PHYSICAL PHENOMENA IN TIME AND FREQUENCY SCALE

\begin{tabular}{llllc}
\hline \hline \multicolumn{1}{c}{ Feature } & Process & Time scale & Frequency scale & Ref. \\
\hline $\begin{array}{l}\text { Melt pool } \\
\text { solidification } \\
\text { Balling } \\
\text { formation }\end{array}$ & SLM & $1 \mathrm{~ms}$ & $1000 \mathrm{~Hz}$ & {$[32]$} \\
$\begin{array}{l}\text { Melt pool } \\
\text { oscillation }\end{array}$ & EBM & $0.5 \mathrm{~ms}$ & $2000 \mathrm{~Hz}$ & {$[33]$} \\
$\begin{array}{l}\text { Keyhole } \\
\text { formation }\end{array}$ & $\begin{array}{l}\text { Laser } \\
\text { welding }\end{array}$ & $0.5-0.8 \mathrm{~ms}$ & $1200-2000 \mathrm{~Hz}$ & {$[34]$} \\
\hline \hline
\end{tabular}

(typically on the order of 100 to $1000 \mathrm{~mm} / \mathrm{s}$ ) [7]. The relative distance $d_{\text {shot }}$ among two consecutive frames is linked to the camera frame rate $f p s$, according to the following relation:

$f p s=v / d_{\text {shot }}$

A reasonable frame distance to assure a detailed observation of the moving melt pool could be $200 \mu \mathrm{m}$, roughly the melt pool width [37]-[39]. Depending on the scanning velocity employed, a frame rate should be set in order to ensure a timely imaging of the melting dynamics. For example, at scanning velocities of 200 and $400 \mathrm{~mm} / \mathrm{s}$, acquisition rates of 1000 and 2000 fps respectively are needed to assure the desired distance between consecutive frames. Consequently, capturing melt pool dynamics might expect to require a data collection rate in the $\mathrm{kHz}$ range. Furthermore, the exposure time affects the sensor capability to detect dynamic events that could be of interest for the on-going process analysis. In particular, increasing the exposure time implies a reduced temporal information content of the image, since the sensor integrates the incoming radiation over a larger time window. If the incoming radiation is sufficient in exciting the camera sensor, a low exposure time is suitable in order to capture fast dynamic events and assure a high image sharpness [40].

\section{B. Spatial resolution criteria}

The spatial extent of the physical phenomenon under inspection determines the field of view (FOV) and the spatial resolution needed for the detection of geometrical features. The melt pool size strongly depends on process parameters such as laser power or scanning speeds. Melt pools are typically $0.5-1.5 \mathrm{~mm}$ long [25], [33] with a width around 150$200 \mu \mathrm{m}$ [37]-[39]. In order to describe accurately the shape of the melt pool, the spatial resolution should be at least one order of magnitude lower than the minimum characteristic dimension of the physical phenomenon under observation, in this case the melt pool width. Accordingly, a suitable spatial resolution can be fixed at $15 \mu \mathrm{m} / \mathrm{pixel}$. The dimensions of the field of view are set considering the spatial extent of the molten pool and the experimental setup employed. A coaxial setup allows to reduce significantly the FOV dimensions. In fact, instead of visualizing the whole build platform from a lateral perspective, the coaxial implementation of the imaging system via dichroic mirror enables a field of view that moves together with the laser itself. In that way, the FOV can be 
focused only on the process area [7]. Its dimensions should be set considering the maximum characteristic dimension of the physical phenomenon under inspection, i.e. the melt pool length. Since the laser beam can move in any direction, the melt pool tail can be oriented in any direction in space. Accordingly, the field of view in a coaxial setup should be large enough to accommodate at least twice the melt pool length and to catch variations of the melt pool size. Accordingly, a $4.5 \mathrm{~mm}$ square FOV can be chosen, discretized in $300 \times 300$ pixels to have a spatial resolution of $15 \mu \mathrm{m} /$ pixel. The final configuration is summarized in Fig. 3. The data rate related to the designed parameters is below the limit associated with the real-time data transfer. In fact, assuming a frame rate of $2000 \mathrm{fps}, 300 \times 300$ pixels and a bit depth of 8 bit, the data rate is $1.44 \mathrm{Gbit} / \mathrm{s}$ (Eq. 3). Despite the significant data rate involved, a real-time data transport is possible using common digital interfaces.

\section{Wavelength band selection criteria}

The wavelength band of interest should be set according to process emission properties. Plume and ionized gas can emit in the UV-visible range [31]. Another interesting wavelength band is the IR region, in which most of the thermal radiation is emitted at typical SLM melting temperatures. Consequently it can be suitable for monitoring the heating and cooling behavior of the processed material [41]. Furthermore, the choice of the observation bands must consider the sensitivity of the sensor and the transmission curve of each optical component between the sensor and the object under inspection, usually optimized for laser wavelength $(1070 \mathrm{~nm})$, in order to assure an effective process band observation. Although the spectral emission can be exploited to gather information on the status of the ongoing process, the emission from the process itself prevents a direct observation of the surface where laser-material interaction takes place, since it acts as a non-stable light source both spatially and temporally. The surface structure and the real melt pool geometry can be visualized by employing a monochromatic external light illuminating the processed area. By placing a narrowband filter centered on the illuminator wavelength just in front of the camera, the broadband emission contribution is reduced and the camera sees the predominant intensity distribution of the reflected external light, thus imaging the processed surface [40].

\section{EXPERIMENTAL SETUP AND Monitoring Module}

\section{A. Selective laser melting system}

A SLM machine prototype, called Powderful, was used in this work [13]. It allowed to process small powder quantities $(<0.5 \mathrm{~kg})$ with an available workspace area of $60 \times 60 \times 20 \mathrm{~mm}^{2}$. The whole process was operated in a closed chamber where an inert Argon atmosphere was created. A single mode fiber laser with a $250 \mathrm{~W}$ maximum power and an emission wavelength of $1070 \mathrm{~nm}$ (IPG YLR-150/750-QCW-AC, Cambridge, MA, USA) was employed. The laser optical chain was composed of a $50 \mathrm{~mm}$ collimating lens, a two-lens system for regulating the
TABLE II

CHARACTERISTICS OF THE MONITORING MODULE IMPLEMENTED

\begin{tabular}{lll}
\hline \hline \multirow{2}{*}{ Symbol } & \multicolumn{1}{c}{ Description } & \multicolumn{1}{c}{ Value } \\
& & $1200 \mathrm{fps}$ \\
\hline$f p s$ & Frame rate & $29 \mu \mathrm{s}$ \\
$t_{\text {exp }}$ & Exposure time & $8 \mathrm{bit} / \mathrm{pixel}$ \\
$b d$ & Pixel bit depth & $304 \times 304$ pixels \\
$R O I$ & Region of interest & $4.3 \mathrm{~mm}$ x $4.3 \mathrm{~mm}$ \\
$F O V$ & Field of view & $14 \mu \mathrm{m} / \mathrm{pixel}$ \\
$r_{s}$ & Spatial resolution & VIS emission $650 \pm 40 \mathrm{~nm}$ \\
$\lambda$ & Wavelength band & NIR emission $850-1000 \mathrm{~nm}$ \\
& & External illumination $640 \mathrm{~nm}$ \\
\hline \hline
\end{tabular}

focal plane position (VarioScan 20, Scanlab, Puchheim, Germany) and a $420 \mathrm{~mm}$ f-theta lens. The resulting beam waist diameter was calculated as $70 \mu \mathrm{m}$. The open architecture allowed a full control and customization of the SLM system and a flexible integration of the optical elements into the existing laser optical chain.

\section{B. Implemented monitoring module characteristics}

The sensor chosen for the implementation was a compact and industrial CMOS camera (Ximea xiQ -USB3 Vision Camera, MQ013xG-ON model). A CMOS sensor was investigated due to its lower cost, smaller pixel size (favorable for achieving high spatial resolution according to (1)) and higher frame rates than comparable infrared cameras. The adoption of a high sensitive CMOS sensor and USB 3.0 interface for data transport together with the possibility to set the active region of pixels resulted in acquisition rates over $1000 \mathrm{~Hz}$. The pixel size was $4.8 \mu \mathrm{m} \times 4.8 \mu \mathrm{m}$. The camera was implemented coaxially via dichroic mirror transparent to the laser radiation and reflective between $400-1000 \mathrm{~nm}$, thus deflecting the process radiation towards the camera sensor. Once the camera sensor has been chosen, the proper optical magnification unit should be implemented according to (1) in order to assure the desired spatial resolution of $15 \mu \mathrm{m} / \mathrm{pixel}$. A lens objective unit with a $120 \mathrm{~mm}$ focal length was added after the dichroic mirror in order to assure the correct magnification of the object image, equal to 0.34 , thus obtaining a final spatial resolution of $14 \mu \mathrm{m} / \mathrm{pixel}$. Accordingly, the number of active pixels was fixed at $304 \times 304$ pixels, corresponding to a FOV equal to $4.3 \mathrm{~mm} \times 4.3 \mathrm{~mm}$ by using (2) and (3), which allowed a maximum frame rate of $1200 \mathrm{fps}$. The resulting data rate calculated with (4) was approximately $887 \mathrm{Mbit} / \mathrm{s}$. Fig. 2 depicts the position of the working point in terms of frame rate, number of pixels employed and resulting data rate, which is shown to be consistent with the melt pool monitoring solutions employed in SLM works. The exposure time of the camera was set at its minimum allowable value, equal to 29 $\mu \mathrm{s}$. All the parameters are summarized in Table II.

A short pass filter at $1000 \mathrm{~nm}$ was placed before the objective unit to prevent the laser back reflection from damaging the imaging lens system and the camera sensor. The wavelength band of interest could be set by interchanging the optical filter just before the camera sensor. The emission from the process was viewed in visible range at about $650 \mathrm{~nm}$ setting a bandpass filter (Thorlabs, FB650-40, Newton, NJ, 


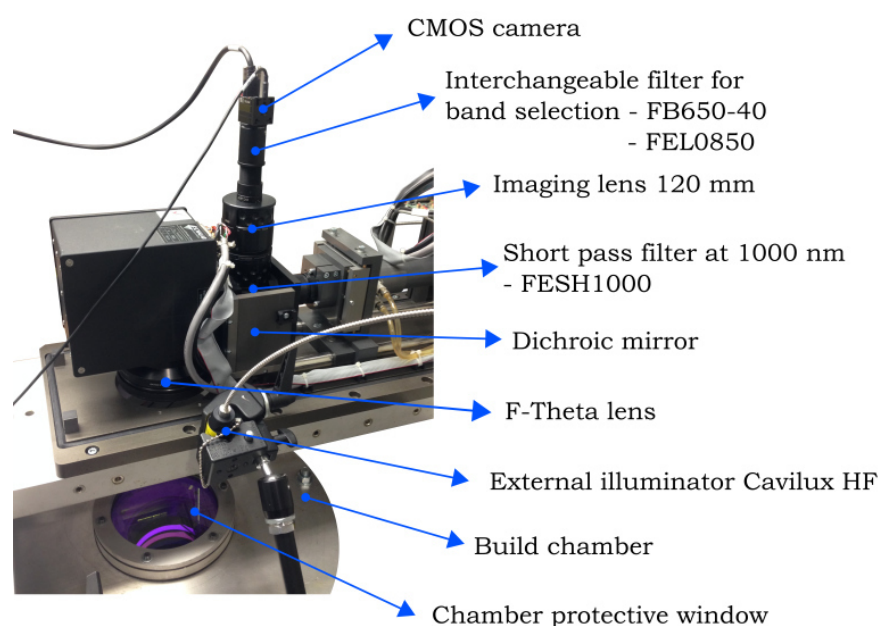

Fig. 4. View of the SLM system with monitoring module.

USA) and in the NIR range between 850-1000 nm employing a long pass filter (Thorlabs, FEL0850, Newton, NJ, USA). The observation of the NIR region is possible with a common silicon-based sensor since CMOS sensors are sensitive in the NIR spectrum up to $1 \mu \mathrm{m}$. Moreover, if the intensity of the emitted radiation is sufficiently high, they allow for a higher frame rate at a lower cost than comparable InGaAs cameras. A narrow band observation was intentionally chosen to avoid chromatic aberrations due to the f-theta lens, which is not designed for imaging purposes. A wider wavelength band was chosen in the NIR range to have a sufficient radiation intensity exciting the CMOS camera sensor, less sensitive outside the visible range. This also compensates for an optical attenuation caused by the galvanometric mirrors, which feature around $80 \%$ transmittance in the observed NIR band. Along with the emission from the process, also the melt pool geometry was investigated directly by using an external illuminator emitting at $640 \mathrm{~nm}$ (Cavitar, Cavilux HF, Tampere, Finland). The external light was synchronized with the camera and a narrowband filter around the illuminator wavelength was employed within the coaxial chain (Thorlabs, FB650-40, Newton, NJ, USA). The illuminator was positioned in an offaxis configuration with respect to the monitoring module. Fig. 4 depicts the open SLM machine with the monitoring module.

\section{Experimental plan}

A specific test geometry was designed, built and monitored, which was composed by a large scanning area $\left(5 \times 5 \mathrm{~mm}^{2}\right)$, denoted in this work as bulk geometry, and two rectangular thin walls $\left(1 \times 10 \mathrm{~mm}^{2}\right)$, as depicted in Fig. 5(a). The scan direction was set parallel to the shorter side of the thin walls. Two test specimens were built, varying the emission mode employed, i.e. CW continuous wave or PW pulsed wave, keeping a fixed energy density input. In the $\mathrm{CW}$ emission regime, energy density $E_{C W}$ is defined as [42]:

$E_{C W}=\frac{P_{\text {peak }}}{h \cdot v_{C W} \cdot z}$

where $P_{\text {peak }}$ represents the power continuously emitted by the $\mathrm{CW}$ laser source and, as visible in Fig. 5(b), corresponds to the (a)

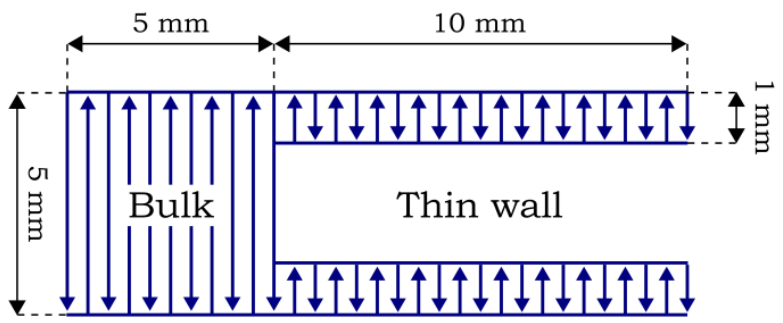

(b)

(c)
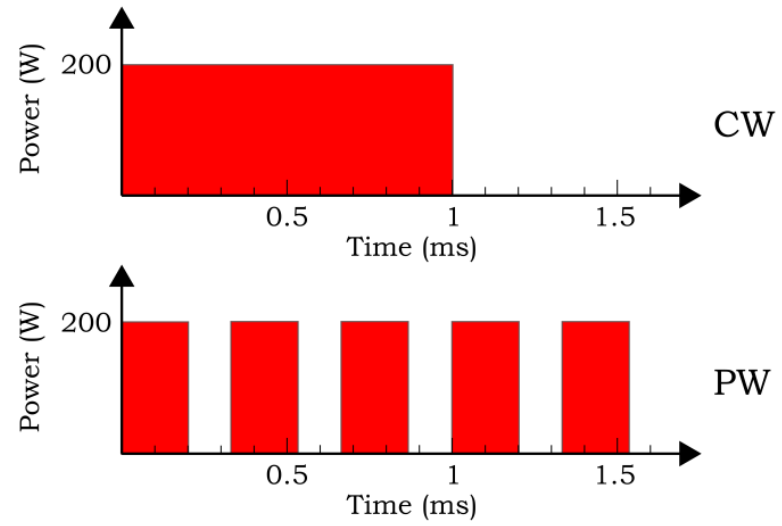

Fig. 5. (a) Test geometry chosen for the experimental campaign. Same energy delivered with different emission modes: (b) $\mathrm{CW}$, (c) PW with $\delta=0.6$, ton $=200$ $\mu \mathrm{s}$.

TABLE III

PROCESS PARAMETERS EMPLOYED IN DIFFERENT EMISSION REGIMES

\begin{tabular}{lcc}
\hline \hline \multicolumn{1}{c}{ Process parameter } & \multicolumn{2}{c}{ Emission mode } \\
& CW & PW \\
\hline Peak power, $P_{\text {peak }}(\mathrm{W})$ & 200 & 200 \\
Average power, $P_{\text {avg }}(\mathrm{W})$ & 200 & 108 \\
Layer thickness, $z(\mu \mathrm{m})$ & 50 & 50 \\
Hatch distance, $h(\mu \mathrm{m})$ & 70 & 70 \\
Energy density, $E\left(\mathrm{~J} / \mathrm{mm}^{3}\right)$ & 143 & 143 \\
Scan speed, $v(\mathrm{~mm} / \mathrm{s})$ & 400 & 216 \\
Duty, $\delta(-)$ & 1 & 0.6 \\
Pulse duration, $t_{o n}(\mu \mathrm{s})$ & $/$ & 200 \\
\hline \hline
\end{tabular}

peak power, $h$ the hatch distance, $v_{C W}$ the scan velocity and $z$ the layer thickness employed. The energy density for PW regime $E_{P W}$ is obtained substituting the power level in the $\mathrm{CW}$ emission with the average power level of the $\mathrm{PW}$ regime $P_{\text {avg }}$ :

$E_{P W}=\frac{P_{a v g}}{h \cdot v_{P W} \cdot z}$

In order to evaluate the effect produced by varying the temporal profile of the laser source at equal energy density, hatch distance, layer thickness and peak power should be maintained at the same level for both emission modes. The concept of varying the temporal emission profile at an equally delivered energy and peak power is illustrated in Fig. 5(b) and (c). As shown, the time required to deliver the same amount of energy increases when modulating laser power. Accordingly, the PW regime requires a lower scan speed to maintain the same energy density. The scan speed in the PW regime can be calculated for a real pulse shape by equaling (6) and (7) [42]:

$v_{P W}=v_{C W} \cdot \frac{P_{\text {avg }}}{P_{\text {peak }}}$ 


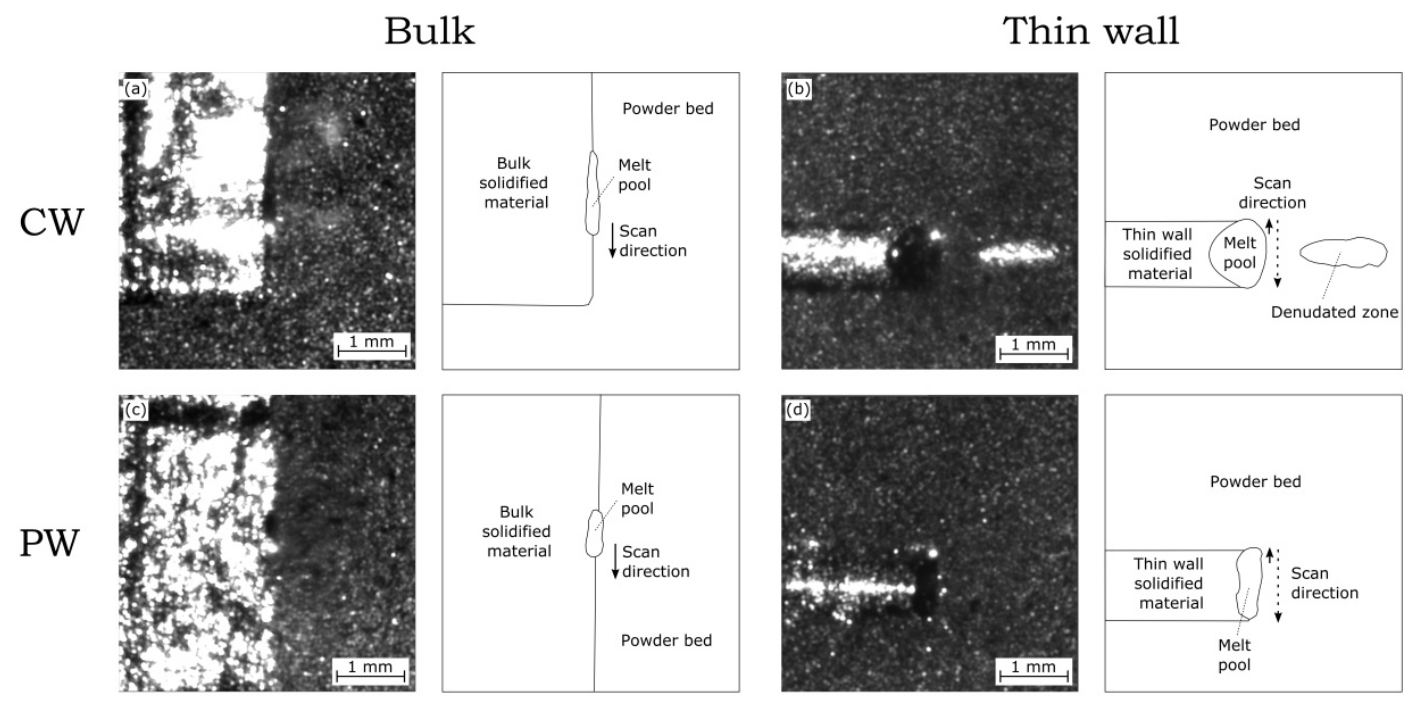

Fig. 6. Externally illuminated images organized according to the part geometry and the emission mode employed, with a schematic representation helping the comprehension of the physical phenomena.

The process parameters were set starting from the $\mathrm{CW}$ regime, where the laser power $P_{\text {peak }}$ was set at $200 \mathrm{~W}$ and scan speed $v_{C W}$ at $400 \mathrm{~mm} / \mathrm{s}$, while for the PW mode a duty cycle $\delta$ of 0.6 and a pulse duration $t_{\text {on }}$ of $200 \mu$ s were selected. In these conditions, the average power in the $\mathrm{PW}$ emission $P_{\text {avg }}$ is measured as $108 \mathrm{~W}$, thus the scan speed $v_{P W}$ was fixed at 216 $\mathrm{mm} / \mathrm{s}$ according to (8) in order to have equal energy density $E$ of $143 \mathrm{~J} / \mathrm{mm}^{3}$. For both regimes, the layer thickness $z$ and hatch distance $h$ were $50 \mu \mathrm{m}$ and $70 \mu \mathrm{m}$ respectively. Through the monitoring system, the emission from the process was viewed in visible and NIR bands as well as reflected external illumination. A summary of the process parameters employed is shown in Table III.

\section{RESUlts}

\section{A. Real melt pool shape from externally illuminated images}

Fig. 6 shows typical images acquired by employing the external light source, with a schematic representation helping the understanding of the physical phenomenon acquired. The melt pool geometry was clearly distinguishable from its dark appearance and occasional bright liquid reflections. An elongated molten pool was visible when scanning bulk zones, while a wide molten area formed when scanning the thin wall geometry. Not only the part geometry, but also the emission mode employed proved to have a large influence on the melting conditions. Even from a visual analysis of external illumination videos, it was possible to note the difference between $\mathrm{CW}$ and PW emission regimes at equal energetic input. The temporal modulation of the laser source resulted in reduced melt pool dimensions for both the bulky and the thin wall geometry. This difference was especially evident in the thin wall zone, where the $\mathrm{CW}$ regime caused a wide molten area, extending to several scan lines, while the PW regime succeeded in keeping a controlled melt pool dimension. Also the powder bed and the highly reflective solidifed material were revealed by the use of an external illumination source. Accordingly, redeposited spatters onto the powder bed or irregularities on the powder distribution could be detected. Fig. 6(b) shows denudated zones, i.e. areas without a correct powder spread, distinguishable thanks to the bright reflections coming from the uncovered solid material. This problem was present in the $\mathrm{CW}$ regime-thin walls zone, and could be linked to the excessive heat accumulation encountered when scanning consequent short vector lengths in a continuous regime [43]. Modulating the laser power with the same level of energy density resulted in a reduced thermal overload, thus avoiding part protrusion from the powder bed.

\section{B. Comparison between visible and NIR wavelength bands}

Fig. 7 shows two distribution maps of the emitted radiance in visible and NIR bands captured while scanning the bulky zone in continuous regime. Looking at the images in visible band, the extraction of the spatial distribution of the emitted radiation resulted in difficulties. While a strong emission was clearly visible in the zone of interaction between the laser and the powder material, the molten pool tail could be extracted with difficulty from the visible emission band. Looking at the Planck spectral radiance of a black body in the neighborhood of typical SLM temperatures around $2000 \mathrm{~K}$ [44], the emitted spectral radiance increased strongly with temperature in visible band. Therefore, the dynamic range of the camera sensor was not sufficient to resolve the intensity distribution, resulting in a saturated zone at higher temperatures where the laser hit the material and non-appreciable intensity values as the molten material cooled down. Conversely, moving towards the IR allowed to resolve the heat distribution in space since the emitted radiance increased more slowly with temperature, thus better exploiting the dynamic range of the camera sensor.

Fig. 8 illustrates images of process emission in the NIR band according to the different combinations of scanned geometry and emission mode employed. The emission shape approximated sufficiently the molten pool geometry observed 
(a)

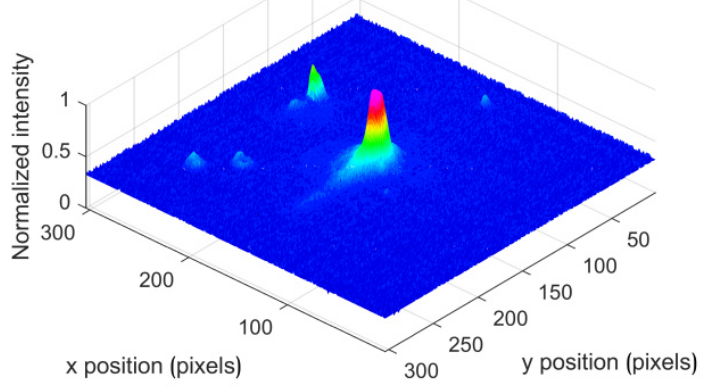

(b)

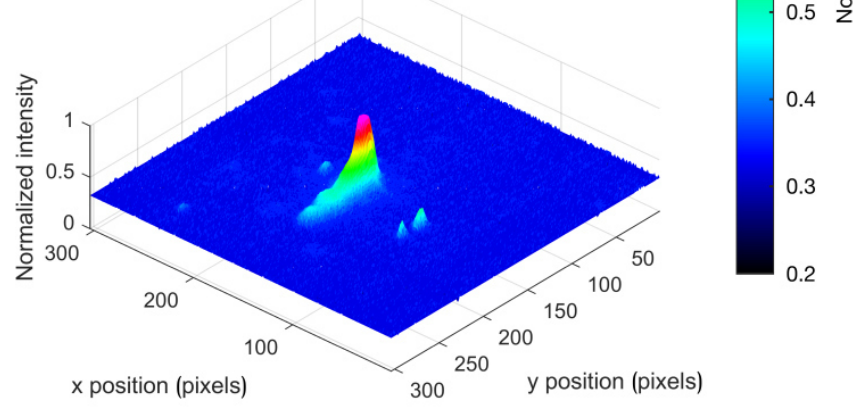

Fig. 7. Intensity distribution map acquired in a) visible and b) NIR band.

in illuminated images. In the bulky geometry, an elongated emission zone was captured, wider in the laser interaction zone and narrower as the laser moved away and the liquid material cooled. The emission region extended when scanning the thin geometry, where the small adjacent scan vector and the surrounding low conductive powder generated an overheating effect. Again, the emission zone narrowed as the laser moved further, with a final shape that resembled the real molten pool geometry observed with the external light. In both scanned geometries, the modulation of the laser power led to a reduced emission area, resulting in a greater control over the thermal load especially in the thin geometry, where the melt pool enlargement may prove to be detrimental for geometrical precision and part density [45], [46], [47]. In addition, spatter ejections were detected by the sensor and they were distinguishable from their small size and fast movement from the melt pool to the outside.

The extraction of the geometrical properties of the molten pool such as length, width and area to gather in-depth information on the course of the melting process is proven to be possible by observing the NIR range. In common pratice, emission images are not physically interpreted, and arbitrary threshold values are set without extracting the real molten pool shape [15], [48]. Other approaches use discontinuities of the acquired gray levels which corresponds to liquidus-solidus transition, succeding in dimensioning the molten area only if a sufficient number of pixels is measuring the intensity gradient [37], [38]. Manara et al. used a probe laser to identify the phase transition zone by detecting the oscillations of the reflected light intensity from the liquid material [49]. Alternatively, temperature measurement can be made by

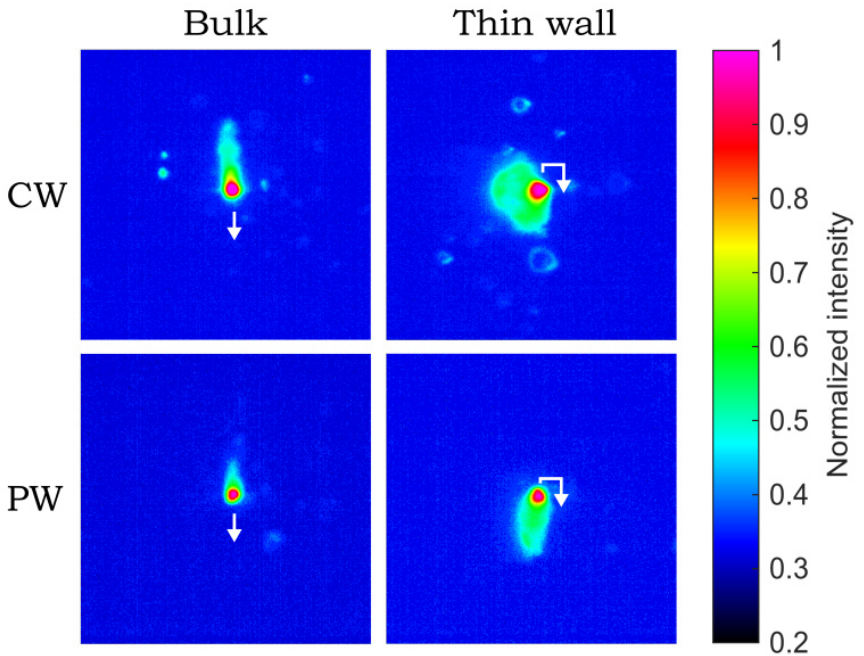

Fig. 8. NIR images acquired in bulk and thin wall geometry when employing different emission modes. The white arrows indicate the beam trajectory.

employing a two wavelength optical setup composed of two cameras [44] or a pyrometer [50]. According to the measured temperature, the molten pool shape can be undirectly estimated. With the monitoring setup hereby developed, it is possible to calibrate the process emission images by comparing them with the real molten area seen through the use of the external light, as reported in [51]. The complementary use of process emission and illuminated images allows to measure the molten pool geometry directly from the NIR emission images, exploiting a simpler image analysis procedure, not possible with illuminated images, and limiting the use of the costly illumination equipment only for calibration purposes.

\section{Observation of the thermal emission behavior in time}

The temporal evolution of process emission in the NIR band can be extracted from the acquired frames. The approach proposed consinsted in the extraction of the intensity distribution along the center of the molten region. By plotting the intensity profile over consecutive frames, a waterfall diagram was obtained, as shown in Fig. 9 for the bulky region. Bearing in mind that the interaction point between laser and material is always centered on the acquired image in a coaxial perspective, each scan line can be identified according to the changing direction of the molten pool tail. A cyclic behaviour in time of the molten pool can be noticed for both the CW and PW regimes. At the beginning of each scan vector, a transient behaviour was observed, with the molten pool length reaching a peak. Then, the molten pool stabilized at an intermediate length up to the end of the scan line, where the molten length decreased. This cyclic behaviour was not related to unpredicted process instabilities, but it was imputed to the dynamic response of the galvanometric mirrors, which featured acceleration and deceleration periods in each scan line. Accordingly, the scan strategy should be improved, adopting a sky writing technique, or the laser power should be adapted in order to keep melting conditions stable. Again, strong differences can be noticed between the $\mathrm{CW}$ and $\mathrm{PW}$ emission modes, not only in terms of the emission length 

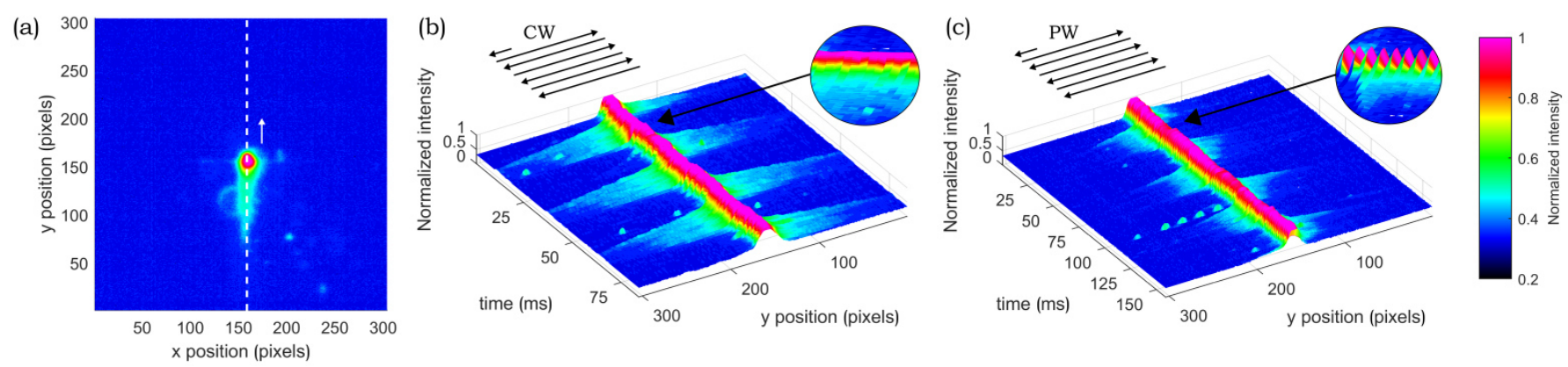

Fig. 9. Intensity distribution plotted over consecutive frames for bulky geometry. a) line chosen for extracting the intensity profile, b) CW map, c) PW map.
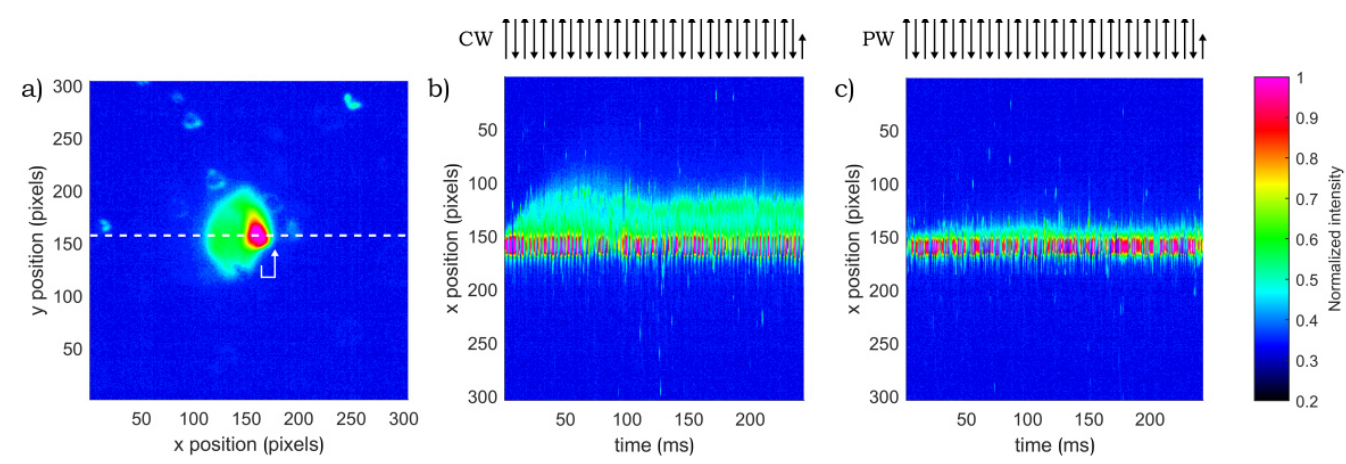

Fig. 10. Intensity distribution plotted over consecutive frames for thin wall geometry. From the left: a) line chosen for extracting the intensity profile, b) CW map, c) PW map.

observed. In fact, a pulsating intensity profile of the lasermaterial interaction can also be extracted, as illustrated in Fig. 9. Finally, ejected particles can be detected and tracked in time as well. Fig. 9(c) shows an example of a molten particle moving along the line chosen for the extraction of the intensity values, distinguishable as a detatched hot particle moving from the molten pool to the outside among consecutive frames.

The same approach was applied to the thin wall geometry, but in this case the intensity profile was extracted perpendicularly to the scan direction in order to extract the evolution of the emission width, as depicted in Fig. 10(a). This was chosen as the representative dimension of process dynamics for the geometry under analysis since the emission length (the dimension parallel to the scan direction) immediately covered the entire thin wall size $(1 \mathrm{~mm})$, while the overheating effect propagated further along the melt pool width. Looking at Fig. 10, a progressive growth of the emission width was encountered when employing the $\mathrm{CW}$ regime. After about $50 \mathrm{~ms}$, corresponding to 17 scanned lines, the emission size stabilized. On the contrary, the PW emission mode featured a smoother temporal trend without an excessive width enlargement, thus succeeding in keeping the thermal load under control in the small geometry.

The proposed solution for the monitoring of the melting conditions in SLM succeeded in acquiring a detailed view of the melting conditions in SLM both in the spatial and temporal domain. Unlike several research approaches that restrict the field of view only to the interaction point of laser and material, thus eliminating the molten pool tail from the imaged area [15], [49], here the field of view was extended to capture the whole molten pool extension and to accommodate its variations in length. The larger field of view implied a higher number of pixels, resulting in a frame rate slightly lower than other literature works, as noticeable in Fig. 2, but still around $1 \mathrm{kHz}$, which resulted to be sufficient for resolving melt pool evolution in time. Of course, higher scan speeds around 2000 $\mathrm{mm} / \mathrm{s}$ encountered in some industrial systems and literature works [52], [53] may require higher acquisition frequencies. Furthermore, the proposed system should be tested and adapted to different powder materials, which are expected to form different molten pool shapes according to the chemical and thermodynamic properties.

\section{Conclusions}

This work presented the design of a monitoring module for melt pool observation in SLM. The choice of the sensor and the imaging optical chain was discussed in order to assure the continuous monitoring of the melting process without limitations on the duration of the recording, but at the same time ensuring a sufficient spatial and temporal resolution to observe the molten pool shape evolution in time. Furthermore, the choice of the emission band was discussed and validated through the use of an external illumination source. The main conclusions are as follows.

- A common CMOS sensor with an adjustable ROI (number of active pixels) in a coaxial configuration was suitable to gather information on the melting conditions, owing to the fast acquisition rate in the $\mathrm{kHz}$ range and the sensor sensitivity up to the NIR band. The only issue was the emitted radiance in the NIR band reaching the 
camera sensor, which should be sufficient to excite the camera sensor at low exposure times. Accordingly, an ad-hoc implementation of the optical chain may be necessary to avoid emitted light losses in the observation band.

- The use of an external illumination proved to be a powerful tool to obtain a direct view of the process conditions and interpret the emission images. Consequently, a more in-depth analysis of emission images was possible, moving towards a physical description of the intensity values acquired, otherwise difficult to realize.

- The NIR band revealed to be more suitable than the visible range to acquire emission from the process. In fact, moving towards the IR allows to better exploit the dynamic range of the camera sensor. Consequently, the spatial distribution of intensity at different temperatures can be acquired.

- The monitoring system employed succeeded in capturing the dynamic behavior of the melting conditions. Time trends captured variations of emission shape, spatter ejections, periodic phenomena and changes in melt pool dimensions when varying the part geometry and the emission regime employed. Consequently, in-depth information on the SLM process physics can be gathered from the monitoring system implemented. Furthermore, the continuous monitoring of the melting conditions, with data available in real time, might lead to the development of a feedback control scheme in the near future.

\section{ACKNOWLEDGMENT}

The authors gratefully acknowledge the collaboration of BLM Group, IPG Photonics Italy and Renishaw.

\section{REFERENCES}

[1] Gong, Haijun, et al. "Analysis of defect generation in Ti-6Al-4V parts made using powder bed fusion additive manufacturing processes." Additive Manufacturing 1 (2014): 87-98.

[2] Kasperovich, Galina, et al. "Correlation between porosity and processing parameters in TiAl6V4 produced by selective laser melting." Materials \& Design 105 (2016): 160-170.

[3] Kempen, Karolien, et al. "Selective laser melting of crack-free high density M2 high speed steel parts by baseplate preheating." Journal of Manufacturing Science and Engineering 136.6 (2014): 061026.

[4] Thompson, Adam, Ian Maskery, and Richard K. Leach. "X-ray computed tomography for additive manufacturing: a review." Measurement Science and Technology 27.7 (2016): 072001.

[5] Bruna-Rosso, Claire, Ali Gökhan Demir, and Barbara Previtali. "Selective laser melting finite element modeling: Validation with high-speed imaging and lack of fusion defects prediction." Materials \& Design 156 (2018): 143-153.

[6] Craeghs, Tom, et al. "Feedback control of Layerwise Laser Melting using optical sensors." Physics Procedia 5 (2010): 505-514.

[7] Berumen, Sebastian, et al. "Quality control of laser-and powder bedbased Additive Manufacturing (AM) technologies." Physics procedia 5 (2010): 617-622.

[8] Bayle, F., and M. Doubenskaia. "Selective laser melting process monitoring with high speed infra-red camera and pyrometer." Fundamentals of laser assisted micro-and nanotechnologies. Vol. 6985. International Society for Optics and Photonics, 2008.

[9] Thombansen, U., and P. Abels. "Process observation in selective laser melting (SLM)." High-Power Laser Materials Processing.
Lasers, Beam Delivery, Diagnostics, and Applications IV. Vol. 9356. International Society for Optics and Photonics, 2015.

[10] Hirvimäki, Marika, et al. "Evaluation of different monitoring methods of laser additive manufacturing of stainless steel." Advanced Materials Research. Vol. 651. Trans Tech Publications, 2013.

[11] Furumoto, Tatsuaki, et al. "Investigation of laser consolidation process for metal powder by two-color pyrometer and high-speed video camera." CIRP Annals-Manufacturing Technology 62.1 (2013): 223-226.

[12] Pavlov, M., M. Doubenskaia, and I. Smurov. "Pyrometric analysis of thermal processes in SLM technology." Physics Procedia 5 (2010): 523-531.

[13] Demir, Ali Gökhan, Chiara De Giorgi, and Barbara Previtali. "Design and Implementation of a Multisensor Coaxial Monitoring System With Correction Strategies for Selective Laser Melting of a Maraging Steel." Journal of Manufacturing Science and Engineering 140.4 (2018): 041003.

[14] Kruth, Jean-Pierre, et al. "Feedback control of selective laser melting." Proceedings of the 3rd international conference on advanced research in virtual and rapid prototyping. 2007.

[15] Fox, Jason C., Brandon M. Lane, and Ho Yeung. "Measurement of process dynamics through coaxially aligned high speed near-infrared imaging in laser powder bed fusion additive manufacturing." Thermosense: Thermal Infrared Applications XXXIX. Vol. 10214. International Society for Optics and Photonics, 2017.

[16] Lane, Brandon, Eric Whitenton, and Shawn Moylan. "Multiple sensor detection of process phenomena in laser powder bed fusion." Thermosense: Thermal Infrared Applications XXXVIII. Vol. 9861. International Society for Optics and Photonics, 2016.

[17] Krauss, Harald, Thomas Zeugner, and Michael F. Zaeh. "Layerwise monitoring of the selective laser melting process by thermography." Physics Procedia 56 (2014): 64-71.

[18] Krauss, H., C. Eschey, and M. Zaeh. "Thermography for monitoring the selective laser melting process." Proceedings of the Solid Freeform Fabrication Symposium. 2012.

[19] Grasso, Marco, and Bianca Maria Colosimo. "Process defects and in situ monitoring methods in metal powder bed fusion: a review." Measurement Science and Technology 28.4 (2017): 044005.

[20] Criales, Luis E., et al. "Laser powder bed fusion of nickel alloy 625 : experimental investigations of effects of process parameters on melt pool size and shape with spatter analysis." International Journal of Machine Tools and Manufacture 121 (2017): 22-36.

[21] Smurov, I. Yu, et al. "Determination of the true temperature during selective laser melting of metal powders based on measurements with an infrared camera." Measurement Techniques 59.9 (2016): 971-974.

[22] Chen, Zhehan, et al. "Image Processing Methods Based on Key Temperature Features for State Analysis and Process Monitoring of Selective Laser Melting (SLM)." 2017 5th International Conference on Mechanical, Automotive and Materials Engineering (CMAME). IEEE, 2017.

[23] Bidare, P., et al. "Fluid and particle dynamics in laser powder bed fusion." Acta Materialia 142 (2018): 107-120.

[24] Matthews, Manyalibo J., et al. "Denudation of metal powder layers in laser powder bed fusion processes." Acta Materialia 114 (2016): 3342.

[25] Bertoli, Umberto Scipioni, et al. "In-situ characterization of laserpowder interaction and cooling rates through high-speed imaging of powder bed fusion additive manufacturing." Materials \& Design 135 (2017): 385-396.

[26] Ly, Sonny, et al. "Metal vapor micro-jet controls material redistribution in laser powder bed fusion additive manufacturing." Scientific reports 7.1 (2017): 4085.

[27] Furumoto, Tatsuaki, et al. "Monitoring of laser consolidation process of metal powder with high speed video camera." Physics Procedia 39 (2012): 760-766.

[28] Krauss, Harald, Thomas Zeugner, and Michael F. Zaeh. "Thermographic process monitoring in powderbed based additive manufacturing." AIP Conference Proceedings. Vol. 1650. No. 1. AIP, 2015.

[29] Calta, Nicholas P., et al. "An instrument for in situ time-resolved Xray imaging and diffraction of laser powder bed fusion additive manufacturing processes." Review of Scientific Instruments 89.5 (2018): 055101.

[30] Toeppel, Thomas, et al. "3D analysis in laser beam melting based on real-time process monitoring." Mater Sci Technol Conf. 2016. 
[31] Spears, Thomas G., and Scott A. Gold. "In-process sensing in selective laser melting (SLM) additive manufacturing." Integrating Materials and Manufacturing Innovation 5.1 (2016): 2.

[32] Li, Yali, and Dongdong Gu. "Thermal behavior during selective laser melting of commercially pure titanium powder: Numerical simulation and experimental study." Additive Manufacturing 1 (2014): 99-109.

[33] Rombouts, Marleen, et al. "Fundamentals of selective laser melting of alloyed steel powders." CIRP Annals-Manufacturing Technology 55.1 (2006): 187-192.

[34] Scharowsky, T., et al. "Melt pool dynamics during selective electron beam melting." Applied Physics A 114.4 (2014): 1303-1307.

[35] Fujinaga, Shigeki, et al. "Direct observation of keyhole behaviour during pulse modulated high-power Nd: YAG laser irradiation." Journal of Physics D: Applied Physics 33.5 (2000): 492.

[36] Perret, O., et al. "Characterization of the keyhole formed during pulsed Nd-YAG laser interaction with a Ti-6Al-4V metallic target." Journal of Applied Physics 90.1 (2001): 27-30.

[37] Heigel, Jarred C., and Brandon M. Lane. "Measurement of the melt pool length during single scan tracks in a commercial laser powder bed fusion process." Journal of Manufacturing Science and Engineering 140.5 (2018): 051012.

[38] Cheng, Bo, et al. "Infrared thermal imaging for melt pool analysis in SLM: a feasibility investigation." Virtual and Physical Prototyping 13.1 (2018): 8-13.

[39] Caprio, Leonardo, Ali Gökhan Demir, and Barbara Previtali. "Comparative study between $\mathrm{CW}$ and PW emissions in selective laser melting." Journal of Laser Applications 30.3 (2018): 032305.

[40] Mazzoleni, L., et al. "External Illumination Strategies for Melt Pool Geometry Monitoring in SLM." JOM (2018): 1-10.

[41] Meola, Carosena, and Giovanni M. Carlomagno. "Recent advances in the use of infrared thermography." Measurement science and technology 15.9 (2004): R27.

[42] Demir, Ali Gökhan, et al. "Complementary use of pulsed and continuous wave emission modes to stabilize melt pool geometry in laser powder bed fusion." Optics \& Laser Technology 113 (2019): 15-26.

[43] Craeghs, Tom, et al. "Determination of geometrical factors in Layerwise Laser Melting using optical process monitoring." Optics and Lasers in Engineering 49.12 (2011): 1440-1446.

[44] Hooper, Paul A. "Melt pool temperature and cooling rates in laser powder bed fusion." Additive Manufacturing 22 (2018): 548-559.

[45] Demir, Ali Gökhan, Paolo Colombo, and Barbara Previtali. "From pulsed to continuous wave emission in SLM with contemporary fiber laser sources: effect of temporal and spatial pulse overlap in part quality." The International Journal of Advanced Manufacturing Technology 91.5-8 (2017): 2701-2714.

[46] Clijsters, Stijn, et al. "In situ quality control of the selective laser melting process using a high-speed, real-time melt pool monitoring system." The International Journal of Advanced Manufacturing Technology 75.5-8 (2014): 1089-1101.

[47] Caprio, Leonardo, Ali Gökhan Demir, and Barbara Previtali. "Influence of pulsed and continuous wave emission on melting efficiency in selective laser melting." Journal of Materials Processing Technology 266 (2019): 429-441.

[48] Thombansen, U., and Peter Abels. "Observation of melting conditions in selective laser melting of metals (SLM)." High-Power Laser Materials Processing: Lasers, Beam Delivery, Diagnostics, and Applications V. Vol. 9741. International Society for Optics and Photonics, 2016.

[49] Manara, D., et al. "New techniques for high-temperature melting measurements in volatile refractory materials via laser surface heating." Review of Scientific Instruments 79.11 (2008): 113901.

[50] Otte, D., H. Kleinschmidt, and O. Bostanjoglo. "Space and time resolved temperature measurements in laser pulse-produced metal melts." Review of scientific instruments 68.6 (1997): 2534-2537.

[51] M. Pacher, L. Mazzoleni, L. Caprio, A. G. Demir, and B. Previtali, "Estimation of melt pool size by complementary use of external illumination and process emission in coaxial monitoring of selective laser melting." 37th International Congress on Applications of Lasers \& Electro-Optics, 2018, pp. 1-10.

[52] Kamath, Chandrika, et al. "Density of additively-manufactured, 316L SS parts using laser powder-bed fusion at powers up to $400 \mathrm{~W}$." The International Journal of Advanced Manufacturing Technology 74.1-4 (2014): 65-78.
[53] Yap, C. Y., C. K. Chua, and Z. L. Dong. "An effective analytical model of selective laser melting." Virtual and Physical Prototyping 11.1 (2016): 21-26.

[54] Tenner, Felix, et al. "Experimental approach for quantification of fluid dynamics in laser metal welding." Journal of Laser Applications 27.S2 (2015): S29003.

[55] Eriksson, Ingemar, John Powell, and A. F. H. Kaplan. "Measurements of fluid flow on keyhole front during laser welding." Science and Technology of Welding and Joining 16.7 (2011): 636641.

[56] Tenner, Felix, et al. "Analysis of the correlation between plasma plume and keyhole behavior in laser metal welding for the modeling of the keyhole geometry." Optics and Lasers in Engineering 64 (2015): 32-41.

[57] Samarjy, Ramiz SM, and Alexander FH Kaplan. "Imaging of the dynamic melt movement induced by a pulsed laser." Physics Procedia 83 (2016): 1308-1318.

[58] Stache, Nicolaj C., et al. "Approaches for high-speed melt pool detection in laser welding applications." Proc. VMV. 2006.

[59] Lane, Brandon, et al. "Thermographic measurements of the commercial laser powder bed fusion process at NIST." Rapid prototyping journal 22.5 (2016): 778-787.

[60] Wegner, A., and G. Witt. "Process monitoring in laser sintering using thermal imaging." SFF Symposium, Austin, Texas, USA. 2011.

[61] Regaard, B., W. Fiedler, and S. Kaierle. "Error detection in lap welding applications using on-line melt pool contour analysis by coaxial process monitoring with external illumination." Lasers in Manufacturing (2007): 471-476.

[62] Ungers, Michael, et al. "In-situ quality monitoring during laser brazing." Physics Procedia 5 (2010): 493-503.

[63] Dorsch, Friedhelm, et al. "Online characterization of laser beam welds by NIR-camera observation." High-Power Laser Materials Processing: Lasers, Beam Delivery, Diagnostics, and Applications II. Vol. 8603. International Society for Optics and Photonics, 2013.

[64] Wen, Peng, Yongqiang Zhang, and Wuzhu Chen. "Quality detection and control during laser cutting progress with coaxial visual monitoring." Journal of Laser Applications 24.3 (2012): 032006.

[65] Haferkamp, Heinz, Martin Goede, and Alexander von Busse. "Quality monitoring and assurance for laser beam cutting using a thermographic process control." Optical Measurement Systems for Industrial Inspection. Vol. 3824. International Society for Optics and Photonics, 1999.

[66] Sichani, E. Fallahi, et al. "Monitoring and adaptive control of $\mathrm{CO} 2$ laser flame cutting." Physics Procedia 5 (2010): 483-492.

[67] Kleszczynski, Stefan, et al. "Error detection in laser beam melting systems by high resolution imaging." Proceedings of the twenty third annual international solid freeform fabrication symposium. Vol. 2012. 2012.

[68] Foster, B., et al. "Optical, layerwise monitoring of powder bed fusion." Solid Freeform Fabrication Symposium, Austin, TX, Aug. 2015 . 